\title{
Doppler Imaging of Close Binaries
}

\author{
Klaus G. Strassmeier \\ Astrophysical Institute Potsdam (AIP), Potsdam, Germany \\ email: kstrassmeier@aip.de
}

\begin{abstract}
Many of the interesting spotted stars are in close binaries where one can find almost any rotational period due to the rotational synchronization with the orbital motion. Binaries are thus good laboratories to study the impact of particular astrophysical parameters that nature usually does not make easily observable. On rapidly-rotating stars, we can indirectly resolve the surface by tomographic imaging techniques and map the surface temperature distribution as a proxy of the (predominantly radial) magnetic field. Binaries are not as straightforward to map as single stars and I will show some examples where it was successful and some where it failed. Eclipses may give some clues on the amount of unresolved features in the images. I present one case of a bright giant of $100 \mathrm{~L}_{\odot}$ in a close binary with even a deformed surface geometry but otherwise solar-type behavior. One of the basic goals is to learn about the impact of interbinary magnetic fields on the evolution of its components in general and to eventually provide conclusive constraints for numerical MHD models on the other hand.
\end{abstract}

Keywords. stars: activity, stars: binaries: close, stars: spots, stars: rotation, techniques: spectroscopic, techniques: polarimetric

\section{Introduction and motivation}

Stellar activity is a collective term for all phenomena inside and outside of a star that are related to its magnetic field, e.g., the rise of flux tubes in a stellar convection zone and their appearance as spots and plages on the stellar surface, or magnetically induced particle acceleration and its braking effect on the stellar rotation, or simply the interplay between a magnetic field and its surrounding plasma. The magnetic field plays the key role in many astrophysical processes but, partly because its mathematical treatment can be somewhat complicated, it is usually the first process that is neglected in evolutionary and other models.

A most important ingredient for our understanding of the solar-stellar connection is the interaction of the magnetic field with the astrophysical plasma. The magnetic field produces the pressure $B^{2} / 8 \pi$ (in cgs units) perpendicular to the field lines and a tension $B^{2} / 4 \pi$ along the field lines but since charged particles move freely parallel to the field, the plasma is not affected by the magnetic tension. For example, in a young sunspot horizontal equilibrium is reached when the difference of the gas pressure outside and inside the spot equals its magnetic pressure while the vertical equilibrium is basically given by the hydrostatic equilibrium. The plasma in a sunspot reaches a stable equilibrium by lowering its vertical position (the so-called Wilson depression) because the pressure in its surrounding increases with depth. Of course, spots can sink because they are cooler than their surrounding. If the magnetic pressure exceeds the gas pressure a current must flow along the field lines.

Therefore, a stellar magnetic field is intimately related to flows, most notably due to stellar rotation and differential rotation. The quest to understand stellar magnetic activity is thus also always a quest to understand stellar rotation and stellar differential 
rotation. In Doppler imaging, one uses the fact that stars rotate in order to map their surface inhomogeneities and to detect, e.g., differential surface rotation.

Several reviews appeared on the subject of "magnetic activity of binary stars", e.g., Guinan \& Giménez (1993) or Strassmeier (2002). A more recent review on the subject of Doppler imaging of binaries is Strassmeier (2005) but see also the many papers in the "Bruxelles" proceedings (Boffin et al. 2001). In the present paper I will emphasize the technical requirements for Doppler and Zeeman-Doppler imaging and update on the literature of binary-star results.

\section{The technique of Doppler imaging}

\subsection{Principle}

Doppler imaging (DI) is a computational technique similar to medical tomography that inverts a series of high-resolution spectral line profiles into an "image" of the stellar surface (Deutsch 1958, Vogt et al. 1987, Rice et al. 1989, Strassmeier 1990, Collier Cameron 1992, Piskunov \& Rice 1993, Berdyugina et al. 1998, Rice \& Strassmeier 2000, Rice 2002). Cool starspots produce distortions in the spectral line profiles that systematically change during a star's rotation. It is the way in which these distortions change with time which allows to reconstruct the stellar surface temperature distribution. The technique is very similar to that described by Richards (2007) in these proceedings except that the velocity range in the line profile is restricted to the (rotating) stellar surface and the intensity range is given by the temperature contrast between spotted and unspotted photosphere.

\subsection{Observational requirements}

Two stellar parameters - brightness and rotational broadening — dictate the instrumental requirements for Doppler imaging. The former sets a limit for the achievable $\mathrm{S} / \mathrm{N}$ ratio and the latter determines the size of a resolution element across the stellar surface. So far, current telescope and spectrograph combinations limited Doppler imaging to stars with $v \sin i$ as low as $17.5 \mathrm{~km} \mathrm{~s}^{-1}$ (Strassmeier \& Rice 1998; using the CFHT and its Gecko spectrograph on EK Dra, G1.5V, at $R \approx 120,000$ ) and $18 \mathrm{~km} \mathrm{~s}^{-1}$ (Washuettl \& Strassmeier 2001; using ESO's CAT and CES for AG Dor, K0V+K4V, at $R \approx 50,000$ ) and $V$ as faint as $\approx 14.3 \mathrm{mag}$ (Strassmeier et al. 2005; using ESO's VLT and UVES on MN Lupi, M0, at $R \approx 60,000$ ). Least-squares deconvolution techniques (Donati et al. 1997) may eventually push the brightness limit several magnitude further.

\subsection{Computational requirements}

As an example, our DI code TEMPMAP (e.g. Rice 2002) performs either single-line, multi-line or full-spectrum inversion in integral light. A comparison of these modes was presented in a poster at the Cool Star 14 workshop (Rice \& Strassmeier 2007). In any mode the code solves the equation of transfer at 72 depth points on 2592 surface elements through a set of 10 Kurucz ATLAS-9 or 12 model atmospheres. Either the default mixinglength description of convective flux or the non-parameterized convective-flux description of Canuto \& Mazzitelli (1992) may be used. We found generally good agreement with local line profiles computed from either of them (see Strassmeier \& Rice 1998). Our code uses either Maximum Entropy or a Tikhonov regularization and can also include two-bandpass photometry in its solution simultaneously. Atomic line data must be known accurately and we usually adopt transition probabilities from VALD (Kupka et al. 1999), or use our own values. The computing demand increases with the number of spectral lines, its sampling - in phase as well as in wavelength - its number of surface elements and the number of model atmospheres and their depth points. Generally speaking, a high-end 

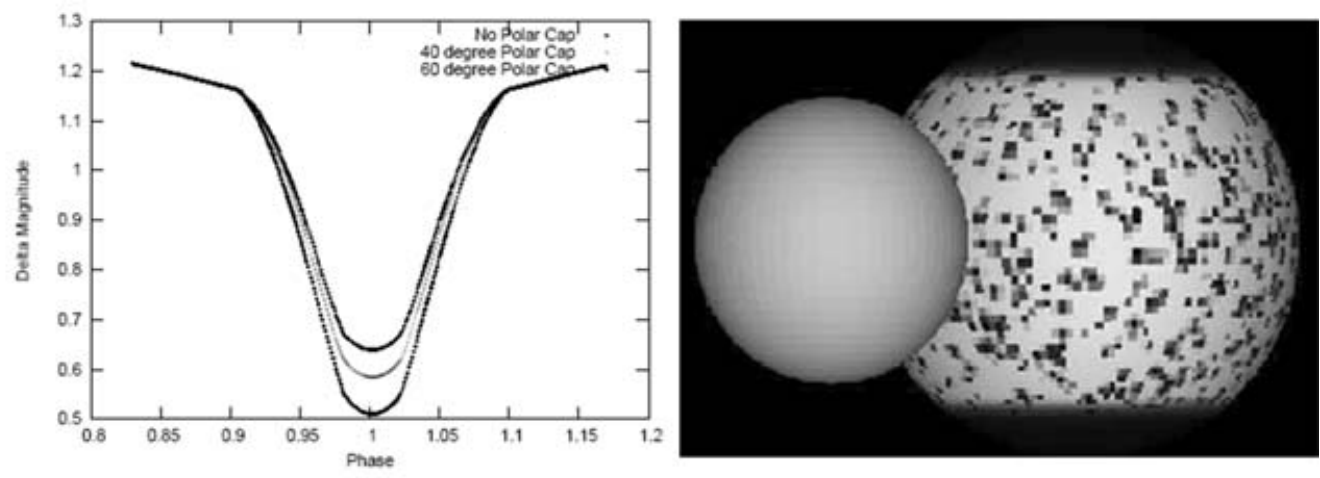

Figure 1. Left: The effect of a polar spot on the primary eclipse light curve of SV Cam. Right: Visualization of SV Cam from eclipse mapping. The primary star (the larger star) has two large cap-like polar spots and is peppered with unresolved spots amounting to a filling factor of alone $28 \%$. Adapted from Jeffers et al. (2006).

PC can handle a single-line inversion with, say, ten blends in 10 minutes. A small 4-8 CPU Beowulf-type cluster is needed to run the full-spectrum mode.

Atomic-line contamination by molecular lines (mostly $\mathrm{TiO}, \mathrm{OH}, \mathrm{CO}, \mathrm{FeH}$, etc.) becomes an issue even at effective temperatures as high as the Sun's. New codes that take into account molecular bands now exist (Berdyugina 2002, Savanov \& Strassmeier 2005) but did not yet lead to published results. The computing demands become significant but can still be handled on a single high-end PC.

The inversion process for Zeeman Doppler imaging (ZDI) requires particularly extensive calculations of local Stokes profiles over the entire stellar disk. Usually these data are pre-tabulated but in case of ZDI become unrealistically large due to an arbitrarily complex magnetic-field structure. Also, the weak-field approximation does not provide the needed accuracy, besides that it is only applicable for Stokes V. Forward numerical tabulation of the integration of the polarized radiative transfer equation becomes therefore a numerically not manageable task. Kopf et al. (2007) have developed an approximation method based on Multi Layer Perceptrons (MLP), a common type of artificial neural networks, that uses the decomposition of local Stokes profiles into their eigenspectra via a Principal Component Analysis. The adaptation (training) of the MLPs is based on a conventional numerical integration of the polarized radiative transfer with the quadratic DELO method. After training with tens of thousands of line profiles, the MLP method yields a speed-up of a factor 1,000 compared to DELO (e.g., Kochukhov \& Piskunov 2007). Calculating the Stokes V profile with the MLP method yields an error of $0.18 \%$, while the weak-field approximation gives an error of $2.3 \%$ (Kopf et al. 2007). Computations currently require a medium-size PC cluster like our own 320-PC 700 Gflops/s machine.

\section{Some applications to close binary stars}

In the following, I will discuss some of the results on a few selected binary systems.

\subsection{SV Cam: an eclipsing SB2 binary with a hot active component}

Jeffers et al. (2006) obtained spectrophotometric eclipse light curves with HST/STIS and mapped the primary F9 component of this F9V+K4V-IV binary ( $P_{\text {orb }}=0.593$ days). They found that the observed surface flux from the eclipsed low-latitude regions of the 

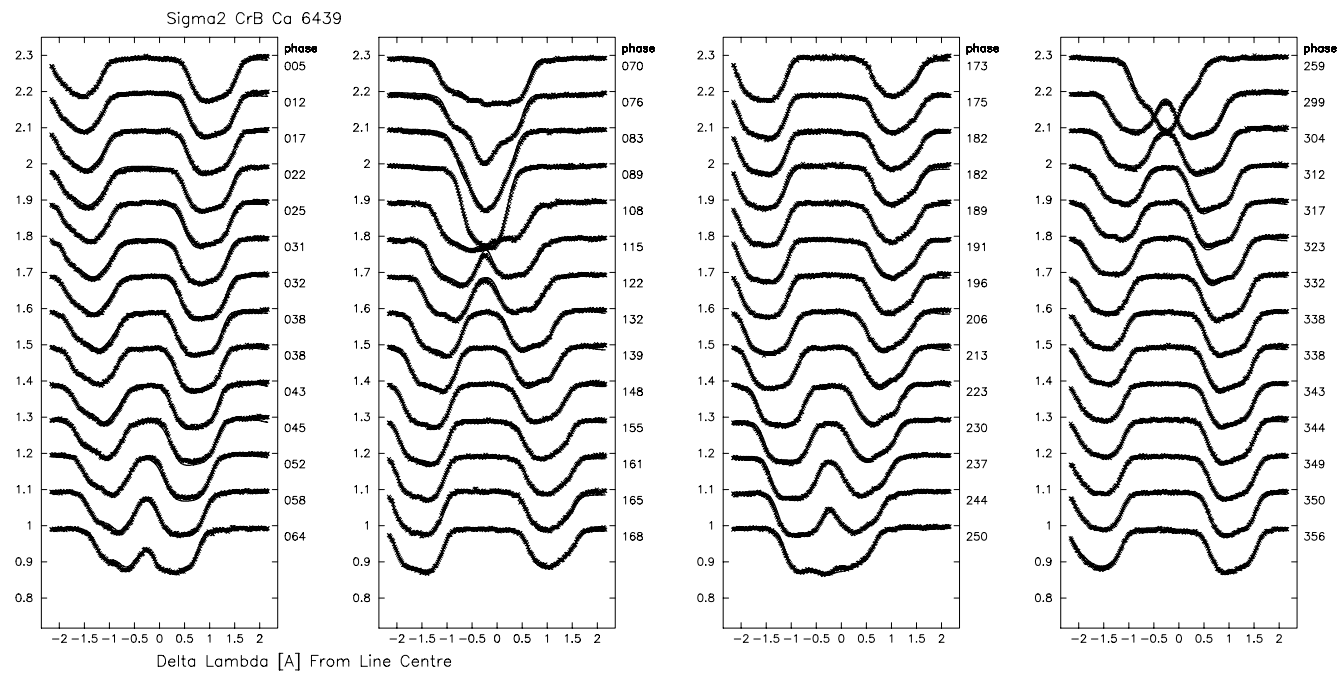

Figure 2. CFHT data for Doppler imaging of $\sigma^{2} \mathrm{CrB}$. Observations and fits for both stellar components are shown from the inversion of the CaI $643.9-\mathrm{nm}$ region. The inversion is done simultaneously for both components with orbital parameters fxed. Adapted from Strassmeier \& Rice (2003).

F9 primary was 30\% lower than what is predicted from PHOENIX model atmospheres and the Hipparcos parallax, despite that the eclipse light-curve shape did not reveal spatial surface inhomogeneities. This could be explained only if there is about a third $(28 \%)$ of the eclipsed region covered by unresolved cool starspots. Even if this "dark" spot component is assumed to also exist over the rest of the surface, a huge cap-like polar spot down to a latitude of $( \pm) 48 \pm 6^{\circ}$ was required in order to fit the eclipse light curves (Figure 1, Jeffers et al. 2006). This is to be seen in contrast to the earlier DI results of Lehmann et al. (2002) who found no polar-cap like spot but only high-latitude spots. On the other hand, spot coverage and temperatures from differential TiO-band modelling had indicated spot filling factors of the order of 30-50\% (O'Neil et al. 1998), usually more than what DI from optical spectral line profiles had been given. However, DI of eclipsing binaries is a particularly ill-posed situation due to the north-south mirroring of the Doppler signal and, moreover, the presence of two components in the combined spectrum. Nevertheless, we conclude that SV Cam had shown us a problem that we need to address in future applications.

\subsection{The $F / G$-type $Z A M S$ binary $\sigma^{2} \mathrm{CrB}$}

This particular target is a nice example of a close binary of two solar-type overactive stars $(\mathrm{F} 9 \mathrm{~V}+\mathrm{G} 0 \mathrm{~V})$ that were spun up due to spin-orbit coupling $\left(P_{\mathrm{orb}}=1.14\right.$ days $)$. Single stars with such properties usually do not exist. Both stars resemble the primary of SV Cam (see above). A Doppler image was presented by Strassmeier \& Rice (2003). In this application we have extended TEMPMAP to invert the combined spectrum from both stars simultaneously over a large wavelength range. The full-spectrum solution considered a total of 171 spectral lines and two continuum bandpasses. As for the single-line maps, a total of 56 spectra from all phases was used. Figure 2 shows the entire input data for one spectral-line region.

The $\sigma^{2}$ CrB surface structure exhibits a complex symmetry within the two stellar components. Both component's high-latitude spots appear asymmetric with respect to their rotational poles and tend to anti-face each other with respect to the apsidal line. 

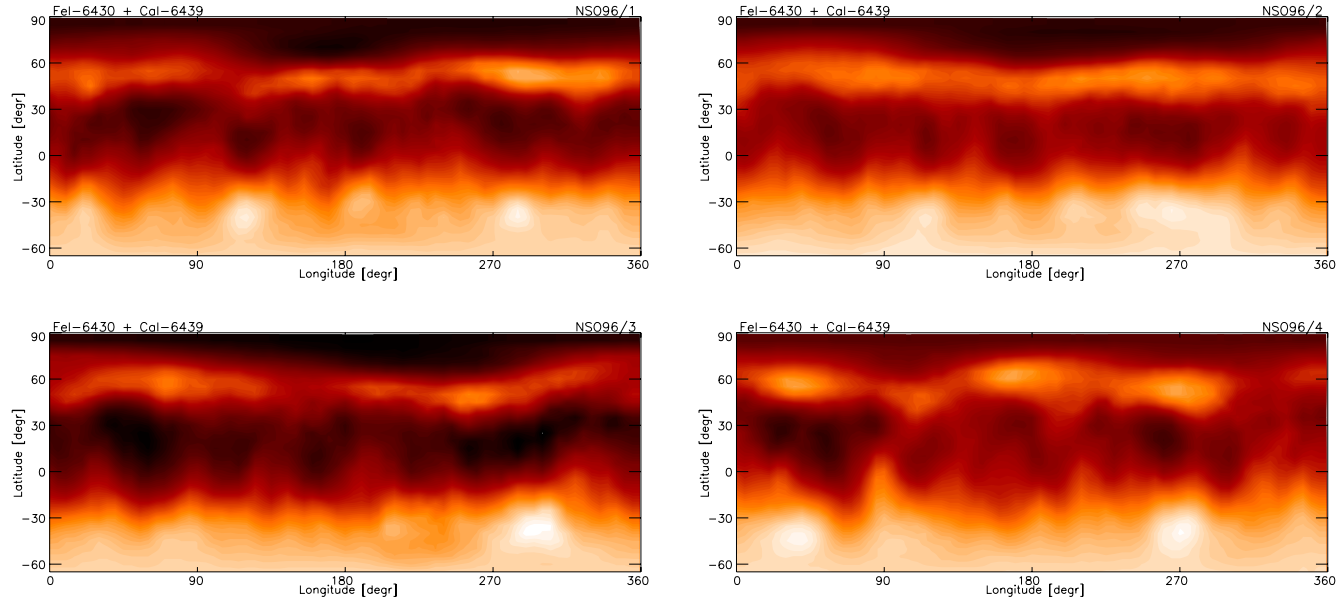

Figure 3. Doppler images of $\zeta$ And for four consecutive stellar rotations in 1996. The maps show temperature between $4800 \mathrm{~K}$ (white) and $3400 \mathrm{~K}$ (black) with spots at high-latitude and even polar spots with temperatures of about $800-1200 \mathrm{~K}$ below the effective photospheric temperature. Data from Kövari et al. (2007b).

A large equatorial warm belt seems to exist on the trailing hemisphere of each of the two stars with respect to the orbital motion, i.e., they appear centered at the central meridian during the respective quadrature phases, and thus are not facing each other as would be the case for a hypothetical irradiation effect. The leading hemispheres on both components appear with an effectively lower temperature than the trailing hemispheres.

We believe that a meaningful interpretation must involve a strong coupling of the individual stellar magnetic fields and its associated electron acceleration and braking along particular field lines. Whether such magnetic coupling in tidally locked stars has an impact on the dynamo and the formation and rise of flux tubes remains to be determined but is suggested from the current results.

\subsection{The ellipsoidal K-giant binary $\zeta$ And}

The star is a magnetically active $\approx 100 \mathrm{~L}_{\odot} \mathrm{K} 1$ giant (with an unseen companion) with its rotation synchronized to the 17.8-day orbital period. Its lithium abundance of $\log n=1.2$ places it in the vicinity of Li-rich RGB stars but it is nevertheless a Li-normal chromospherically active binary star. The primary seems to undergo its first standard dredge-up dilution. The unprojected equatorial rotational velocity, and the fact that the rotational period appears synchronized to the orbital period, suggests a likely radius of the primary of $16.0 \pm 0.2 \mathrm{R}_{\odot}$. The mass function a.o. parameters together with the most likely inclination from DI suggests a $0.75-\mathrm{M}_{\odot} \mathrm{K}$-dwarf secondary circling the primary at a distance of just 2.7 (primary) stellar radii, i.e., 2.2 stellar radii above its surface, giving rise to a tidally deformed primary-star surface.

Kövari et al. (2007b) presented an extensive DI study of this system. Our DI code TEMPMAP had to be modified to work with gravitationally deformed surfaces. Kövari et al. (2007a) (in this proceeding) discusses various tests that were performed during the line-profile inversions of $\zeta$ And and I refer to this paper for more details.

Our Doppler maps revealed cool high-latitude and even polar spots with temperatures of about 800-1200 K below the effective photospheric temperature (Figure 3 ). The polar spot faded by several hundred degrees within one to two stellar rotations during at least one occasion in 1996/97. It was recovered with the previous contrast (1200 K) one year 
later though. The low-latitude spots tended to group on the two hemispheres visible during quadrature, i.e., $\pm 90^{\circ}$ from the apsidal line following and preceding the location of the secondary star. This seemed to be the case for both observing seasons we had data for. At the same time the cool polar spot had a large appendage near the phase of conjunction with the secondary behind in 1996/97, but less determinable in 1997/98. A cross-correlation analysis of consecutive maps and a fit with a solar-type quadratic law revealed a more rapidly rotating equator with a surface shear with respect to higher latitudes of four times lower than for the Sun and with a lap time of 360 days.

\section{Summary}

The sum of the activity phenomena detected up to now is staggering and significantly more complex than what just scaled-up solar analogy would imply. Various groups found

(a) large spots on or near the rotational pole (e.g., Vogt et al. 1999, Strassmeier 1999, Berdyugina et al. 1998, Collier-Cameron 1995),

(b) differential rotation both in the same sense and in the opposite sense than on the Sun (e.g., Vogt et al. 1999, Donati \& Collier-Cameron 1997, Hatzes 1998, Rice \& Strassmeier 1996, Strassmeier et al. 2003, Donati et al. 2003, Marsden et al. 2006), and

(c) seasonal variations thereof (e.g., Donati et al. 2003, Marsden et al. 2007),

(d) possibly detected (meridional) flows toward the pole (Weber \& Strassmeier 2001, Strassmeier \& Bartus 2000, Kövari et al. 2007b, Weber \& Strassmeier 2007),

(e) detected and mapped transiting prominences (Dunstone et al. 2007, Jardine et al. 1998, Collier-Cameron et al. 1996),

(f) warm spots (e.g., Unruh et al. 1998, Strassmeier 1994,1999),

$(g)$ and possibly detected the impact regions from accretion streams in very young pre-main-sequence photospheres (Strassmeier et al. 2005),

(h) observed active longitudes and related activity flip-flops (e.g., Korhonen et al. 2004, Berdyugina 2003, Oláh et al. 2002),

(i) and found good evidence for uniformly distributed and therefore unresolved spots (Jeffers et al. 2006, Hussain et al. 2007),

$(j)$ and found spot evolution on very short- and on very long timescales (e.g. Washuettl et al. 2007, Petit et al. 2004, Barnes et al. 2001) and, finally,

$(k)$ directly detected complex magnetic surface fields (e.g. Donati et al. 2006, Petit et al. 2004, Piskunov \& Kochukhov 2002, Wade et al. 2000). The latter is still a rather virgin field because of the lack of appropriate spectro-polarimetric data.

Finally, after 20 years of Doppler Imaging, Yes, the stars, they always make me laugh.

(A. Saint Exupéry, The Little Prince).

\section{Acknowledgements}

I'd like to thank my colleagues John Rice from Brandon University, and Michi Weber, Thorsten Carroll, Heidi Korhonen and Igor Savanov from AIP for many fruitful discussions on various related software issues.

\section{References}

Afram, N., Berdyugina S.V., Fluri D.M., Suwald, F., Kuhn, J.R., Harrington, \& D.M. 2007, in J. Stauffer et al. (eds.), 14th Cool Stars, Stellar Systems, and the Sun, Pasadena, ASPC, CD-ROM (in press)

Barnes, J.R. \& Collier Cameron, A. 2001, MNRAS, 326, 950 
Berdyugina, S.V. 2002, in Brown A., Harper G., \& Ayres, T. (eds.), 12th Workshop, Cool Stars, Stellar Systems, and the Sun, www-publication, p.210

Berdyugina, S.V. 2003, in Benz, A. \& Dupree, A. (eds.), IAU Symp. 219, Stars as Suns: Activity, Evolution and Planets, Sydney, p.181

Berdyugina, S.V., Berdyugin, A.V., Ilyin, I., \& Tuominen, I. 1998, A\&A, 340, 437

Boffin, H.M.J., Steeghs, D., \& Cuypers, J. (eds.) 2001, Astrotomography, Lecture Notes in Physics, Springer, Vol. 573

Canuto V.M. \& Mazzitelli I. 1992, ApJ, 389, 724

Collier Cameron, A. 1992, in Byrne P.B., \& D.J. Mullan (eds.), Surface Inhomogeneities on Late-Type Stars, Lecture Notes in Physics, Vol. 397, Springer-Verlag, Berlin, p. 33

Collier Cameron, A. 1995, MNRAS, 275, 534

Collier Cameron, A. 1996, in Linsky, J.L., \& Strassmeier, K.G. (eds.), IAU Symp. 176, Stellar Surface Structure, Kluwer, p.449

Deutsch, A. 1958, in Lehnert B. (ed.), Electromagnetic Phenomena in Cosmological Physics, IAU Symp. 6, Cambridge Univ. Press, Cambridge, p. 209

Donati, J.-F. \& Collier Cameron, A. 1997, MNRAS, 291, 1

Donati, J.-F., Forveille, T., Collier Cameron, A., et al. 2006, Science 311, 633

Donati, J.-F., Semel, M. Carter, B.D., Rees, D.E., \& Collier Cameron, A. 1997, MNRAS, 291, 658

Donati, J.-F., Collier Cameron, A., \& Petit, P. 2003, MNRAS, 345, 1187

Dunstone, N., Collier Cameron, A., Barnes, J., \& Jardine, M. 2007, in J. Stauffer et al. (eds.), 14th Cool Stars, Stellar Systems, and the Sun, Pasadena, ASPC, CD-ROM (in press)

Guinan, E.F. \& Giménez, A. 1993, in J. Sahade et al. (eds.), The Realm of Interacting Binary Stars, Kluwer, Dordrecht, p. 51

Hatzes, A.P. 1998, A\& A, 330, 541

Hussain, G.A.J., Saar, S.H., Aufdenberg, J., Ringwald, F., \& Johns-Krull, C. 2007, in J. Stauffer et al. (eds.), 14th Cool Stars, Stellar Systems, and the Sun, Pasadena, ASPC, CD-ROM (in press)

Jardine, M., Barnes, J., Unruh, Y.C., \& Collier-Cameron, A. 1998, in D. Webb et al. (eds.), IAU Colloq. 167, PASPC

Jeffers, S.V., Aufdenberg, J.P., Hussain, G.A.J., Collier Cameron, A., \& Holzwarth, V. 2006, MNRAS, 367, 1308

Kochukhov, O. \& Piskunov, N.E. 2007, these proceedings

Kopf, M., Carroll, T., \& Strassmeier, K.G. 2007, in J. Stauffer et al. (eds.), 14th Cool Stars, Stellar Systems, and the Sun, Pasadena, ASPC, CD-ROM (in press)

Korhonen, H., Berdyugina S.V., \& Tuominen I. 2004, AN 325, 402

Kövari, Zs., Bartus, J., Oláh, K., Strassmeier, K.G., Rice, J.B., Weber, M., \& Forgacs-Dajka, E. 2007a, these proceedings, 212

Kövari, Zs., Bartus, J., Strassmeier, K.G., Oláh, K., Washuettl, A., Weber, M., \& Rice, J.B. 2007b, A\& A, 463, 1071

Kupka, F., Piskunov, N.E., Ryabchikova, T.A., Stempels, H.C., \& Weiss, W.W. 1999, AEAS, 138,119

Lehmann, H., Hempelmann, A., \& Wolter, U. 2002, A\&A, 392, 963

Marsden, S.C., Donati, J.-F., Semel, M., Petit, P., \& Carter, B.D. 2006, MNRAS, 370, 468

Marsden, S.C., Berdyugina, S.V., Carter, B.D., et al. 2007, in J. Stauffer et al. (eds.), 14th Cool Stars, Stellar Systems, and the Sun, Pasadena, ASPC, CD-ROM (in press)

Oláh, K., Strassmeier, K.G., \& Weber, M. 2002, A\&A, 389, 202

O'Neil, D., Neff, J., \& Saar, S.H. 1998, ApJ, 507, 919

Petit, P., Donati, J.-F., Wade, G.A., et al. 2004, MNRAS, 348, 1175

Piskunov, N.E. \& Kochukhov, O. 2002, A\&A, 381, 736

Piskunov, N.E. \& Rice, J.B. 1993, PASP 105, 1415

Rice, J.B. 2002, AN 323, 220

Rice, J.B. \& Strassmeier, K.G. 1996, $A \mathscr{E} A, 316,164$

Rice, J.B. \& Strassmeier, K.G. 2000, $A \& A S, 147,151$

Rice, J.B. \& Strassmeier, K.G. 2007, in J. Stauffer et al. (eds.), $14^{\text {th }}$ Cool Stars, Stellar Systems, and the Sun, Pasadena, ASPC, CD-ROM (in press) 
Rice, J.B., Wehlau, W.H., \& Khokhlova, V.L. 1989, A\&A, 208, 179

Richards, M. 2007, these proceedings, 160

Savanov, I. \& Strassmeier, K.G. 2005, A\&A A, 444, 931

Strassmeier, K.G. 1990, ApJ, 348, 628

Strassmeier, K.G. 1994, $A \mathscr{G} A, 281,395$

Strassmeier, K.G. 1999, A\& $A, 347,225$

Strassmeier, K.G. 2002, in F.C. Lazaro \& M.J. Arevalo (eds.), Selected Topics on Binary Stars: Observations and Physical Processes, Lecture Notes in Physics, Springer Verlag, p.48

Strassmeier, K.G. 2005, in Pavlovski et al. (eds.), Physics of Close Binary Stars, Dubrovník, Croatia, ASPC 318, p.69

Strassmeier, K.G. \& Bartus, J. 2000, A\&A, 354, 537

Strassmeier, K.G., Kratzwald, L., \& Weber, M. 2003, A\&A, 408, 1103

Strassmeier, K.G. \& Rice, J.B. 1998, A\&A, 330, 685

Strassmeier, K.G. \& Rice, J.B. 2003, A\&A, 399, 315

Strassmeier, K.G., Rice, J.B., Ritter, A., Küker, M., Hussain, G.A.J., Hubrig, S., Shobbrook, R. 2005, A\&A, 440, 1105

Unruh, Y.C., Collier-Cameron, A., \& Guenther, E. 1998, MNRAS, 295, 781

Vogt, S.S., Hatzes, A.P., Misch, A.A., \& Kürster, M. 1999, ApJS, 121, 547

Vogt, S.S., Penrod, G.D., \& Hatzes, A.P. 1987, ApJ, 321, 496

Wade, G.A., Donati, J.-F., Landstreet, J.D., \& Shorlin, S.L.S. 2000, MNRAS, 313, 823

Washuettl, A. \& Strassmeier, K.G. 2001, A\& A,, 370, 218

Washuettl, A., Strassmeier, K.G., \& Weber, M. 2007, AN, submitted

Weber, M. \& Strassmeier, K.G. 2001, A\&\&A, 373, 974

Weber, M. \& Strassmeier, K.G. 2007, in J. Stauffer et al. (eds.), $14^{\text {th }}$ Cool Stars, Stellar Systems, and the Sun, Pasadena, ASPC, CD-ROM (in press) 